\title{
Localisation, dénombrement et identification de la contamination microbienne après nettoyage de joints en EPDM d'un circuit de pasteurisation de l'industrie laitière
}

\author{
E Mettler, B Carpentier \\ Laboratoire d'études et de recherches pour l'alimentation collective, \\ Centre national d'études vétérinaires et alimentaires, BP 332, 94709 Maisons-Alfort cedex, \\ France
}

(Reçu le 29 juillet 1996 ; accepté le 9 avril 1997)

\begin{abstract}
Summary - Location, enumeration and identification of the microbial contamination after cleaning of EPDM gaskets introduced into a milk pasteurization line. Microbial contamination after cleaning and wear of gaskets introduced in four places in a milk pasteurization process line were studied during 1-, 4-, 16- and 20-week periods. From the fourth week onwards, gaskets presented signs of wear and fouling, visible to the naked eye and increasing with time. Observation of 20week gaskets using scanning electron microscopy showed a residual fouling depending on processing conditions. Organic fouling was generally higher on the gasket-metal contact area than on the face exposed to circulating fluids. It was made up of closely-associated milk and microbial fouling. Microbial contamination after cleaning was detached using sonication and cells were enumerated by different methods. Acridine orange direct counts showed a stabilized total population beyond $10^{6}$ cells per centimeter of gasket perimeter. The methods used to assess culturable cells showed an increase of this population with time, reaching $10^{3}$ cells per centimeter of gasket perimeter for the four places after 16 or 20 weeks, made up of typical raw milk micro-organisms.
\end{abstract}

gasket / pasteurizer / cleaning-in-place / wear / microbial fouling

Résumé - La contamination microbienne après nettoyage et l'usure de joints placés en quatre points d'une ligne de pasteurisation industrielle de lait sont étudiées pour des durées d'utilisation de $1,4,16$ et 20 semaines. Dès 4 semaines, les joints présentent une usure et un encrassement visibles à l'œil nu, qui s'accentuent par la suite. L'observation par microscopie électronique à balayage des joints de 20 semaines montre un encrassement résiduel dont la nature varie selon leurs conditions d'utilisation. L'encrassement organique, globalement plus abondant dans la zone de contact joint-métal que sur la face exposée aux fluides circulants, est constitué de souillures laitières et microbiennes indissociables. La contamination microbienne après nettoyage, détachée par ultrasons, est dénombrée par plusieurs méthodes. La coloration par l'orangé d'acridine met en évidence une population totale stabilisée au-delà de $10^{6}$ cellules par centimètre de périmètre de joint. Différentes méthodes de 
dénombrement des cellules cultivables montrent une population qui augmente au cours du temps, atteignant $10^{3}$ cellules par centimètre de périmètre de joint pour les quatre emplacements après 16 ou 20 semaines d'utilisation, et qui est constituée de genres caractéristiques du lait cru.

joint / pasteurisateur / nettoyage en place / usure / encrassement microbien

\section{INTRODUCTION}

L'encrassement microbien des parois internes des équipements de l'industrie laitière est encore peu étudié, notamment celui qui affecte les échangeurs de chaleur (Bouman et al, 1982 ; Driessen et al, 1984 ; Langeveld et al, 1995). En effet, en appliquant correctement les barèmes de traitement thermique, on obtient, en principe, la qualité microbiologique recherchée. Autrefois, ce résultat paraissait suffisant, mais aujourd'hui pour maintenir la qualité organoleptique des produits laitiers, les traitements thermiques doivent être limités au juste nécessaire et il faut donc minimiser l'encrassement microbien.

Depuis le début des années 1990 , plusieurs équipes de l'EHEDG (European Hygienic Equipment Design Group) travaillent sur la conception hygiénique des équipements. Leurs travaux ont montré que les efforts à faire en matière d'hygiène se situent au niveau des zones de discontinuité des circuits : les raccords, les vannes, les dispositifs d'échantillonnage, etc. Si l'acier inoxydable est unanimement retenu comme le matériau le mieux adapté à la construction de l'équipement laitier, aucun véritable consensus n' existe à l'heure actuelle sur le matériau le mieux adapté à la réalisation des joints, eux-mêmes reconnus comme points critiques pour la maîtrise de l'hygiène (Curiel et al, 1993). En effet, les joints résistent mal aux traitements qu'ils subissent (Daufin et al, 1981) : agressions par les produits de nettoyage, par la chaleur, par les contraintes mécaniques dues à la dilatation, etc. Ils deviennent rapidement craquelés
(Czechowski, 1990 ; Austin et Bergeron, 1995). Ainsi, si l'on compare des matériaux constitutifs de joints à l'état neuf, ils semblent avoir une aptitude peu différente de celle de l'acier inoxydable à être colonisés par des bactéries (Lewis et Gilmour, 1987 ; Bourion et Cerf, 1996). En revanche leur résistance à l'usure a été étudiée par Curiel et al (1993), qui préconisent les polymères de type nitrile, nitrile/butyl, silicone, et les fluoroélastomères. Austin et Bergeron (1995) observent des bactéries aussi bien sur polymère de type nitrile (Buna- $n^{\circledR}$ ) que sur polytétrafluoroéthylène. D'après Daufin et al (1981), les polymères de types EPDM (copolymère éthylène, propylène, diène, méthylène) et butyl présentent une résistance à l'usure supérieure aux polymères de type nitrile, silicone, styrène-butadiène et chloroprène. Karpinsky et Bradley (1988) montrent également une supériorité de l'EPDM sur le silicone. En revanche, des résultats récents attribuent à l'EPDM une nettoyabilité inférieure à celle du polymère nitrile/butyl (communication personnelle) et à celle de l'acier inoxydable (Wirtanen et al, 1996).

L'encrassement microbien, c'est-à-dire la contamination résiduelle après nettoyage, et l'usure des joints des conduites, n'ont été étudiés, à notre connaissance, que par microscopie en épifluorescence (Czechowski, 1990) et microscopie électronique à balayage (Austin et Bergeron, 1995). L'objectif de la présente étude est de suivre au cours du temps la contamination microbienne après nettoyage et l'usure des joints en EPDM placés en plusieurs points d'un circuit de pasteurisation industriel, pour des 
temps d'utilisation allant jusqu'à 20 semaines. La contamination microbienne après nettoyage comporte une proportion inconnue de cellules viables non cultivables, du fait des agressions subies (Carpentier et Cerf, 1993). C'est pourquoi des méthodes de dénombrement différentes sont utilisées pour caractériser le nombre total de cellules microbiennes et la fraction cultivable, qui est identifiée.

\section{MATÉRIEL ET MÉTHODE}

\section{Joints}

Des joints circulaires en EPDM sont insérés en quatre points du circuit de pasteurisation de lait (pasteurisateur APV R52, ayant un débit de $30000 \mathrm{Lh}^{-1}$ en pasteurisation, et de $33000 \mathrm{Lh}^{-1}$ en nettoyage). Leur emplacement et les conditions d'utilisation correspondantes sont décrits dans le tableau I. Leurs caractéristiques géométriques et leur insertion dans la conduite sont décrites sur la figure 1 . Les joints $\mathrm{A}$ et $\mathrm{P}$ sont identiques à l'état neuf. Les joints ne sont pas en contact avec l'extérieur de la tuyauterie, la jonction étant refermée par un écrou. Les joints sont insérés à l'état neuf dans le circuit, prélevés ensemble avec des outils stérilisés à la fin de leur temps d'utilisation et remplacés par les joints neufs. Différents temps d'utilisation sont étudiés dans l'ordre suivant : $1,20,16$ et 4 semaines. Un seul joint a été récupéré à chaque position et après chaque temps d'utilisation.

\section{Procédures de nettoyage}

Le circuit est nettoyé en place (tableau II) selon des procédures représentatives de celles habituellement appliquées aux échangeurs de chaleur de l'industrie laitière (Kane et Middlemiss, 1985). Les joints sont prélevés après un nettoyage complet. Celui-ci est suivi d'une circulation d'eau à $95^{\circ} \mathrm{C}$ pendant 110 minutes, mais le prélèvement des joints doit avoir lieu avant cette étape, le circuit ne pouvant être ouvert entre celle-ci et la reprise de la production. Les joints sont expédiés au laboratoire dans des sachets individuels stérilisés, en conditionnement réfrigéré, et analysés 24 heures après le prélèvement.

\section{Observation de l'usure et de l'encrassement des joints}

\section{A l'œil nu}

Dès la réception des joints, leur état physique est comparé visuellement à celui des joints neufs correspondants pour les critères suivants : modification géométrique de la section du joint, pré-

Tableau I. Conditions dans lesquelles ont été placés les joints étudiés (dans l'ordre du parcours du lait). Processing environment in the different places where gaskets were introduced (presented following the milk circulation).

\begin{tabular}{|c|c|c|c|c|}
\hline & $A$ & $C$ & E & $P$ \\
\hline Emplacement & Arrivée du lait & $\begin{array}{l}\text { Extrémité du tuyau } \\
\text { central de } \\
\text { chambrage }\end{array}$ & $\begin{array}{l}\text { Sortie de } \\
\text { l'écrémeuse }\end{array}$ & Sortie du lait \\
\hline $\begin{array}{l}\text { Nature du lait } \\
\text { en contact }\end{array}$ & Lait cru & $\begin{array}{l}\text { Lait en cours } \\
\text { de pasteurisation * }\end{array}$ & $\begin{array}{l}\text { Lait écrémé } \\
\text { pasteurisé }\end{array}$ & $\begin{array}{l}\text { Lait écrémé } \\
\text { pasteurisé }\end{array}$ \\
\hline $\begin{array}{l}\text { Température } \\
\text { du lait en contact }\end{array}$ & $2^{\circ} \mathrm{C}$ & $86^{\circ} \mathrm{C}$ & $55^{\circ} \mathrm{C}$ & $2^{\circ} \mathrm{C}$ \\
\hline
\end{tabular}

\footnotetext{
* : a séjourné 15 secondes à $86^{\circ} \mathrm{C}$, soit la moitié de la durée totale de la pasteurisation.
}

* : processed for $15 \mathrm{~s}$ at $86^{\circ} \mathrm{C}$, which is half the total time of pasteurization. 


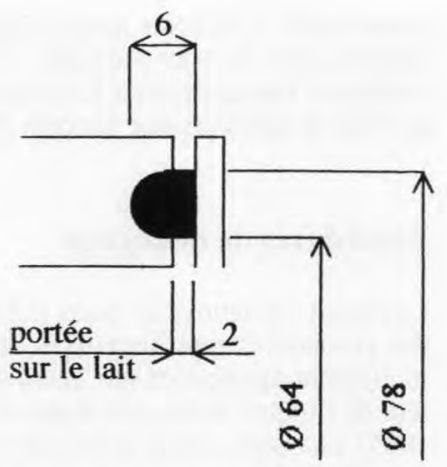

ENTRÉE LAIT CRU (JOINT A)

SORTIE LAIT PASTEURISÉ (JOINT P)

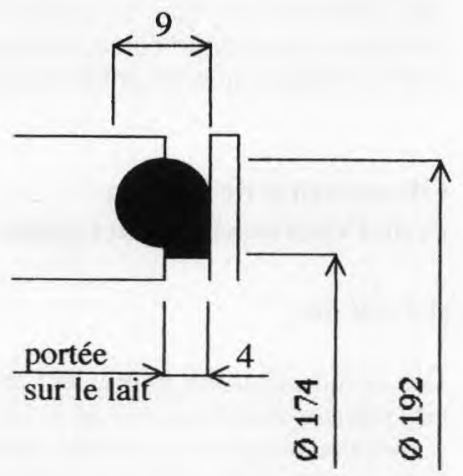

CHAMBREUR (JOINT C)

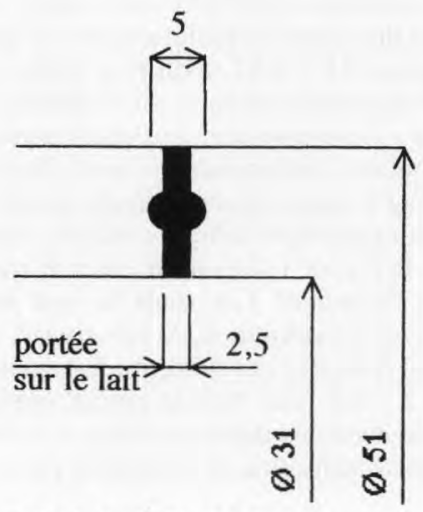

SONDE ÉCREMEUSE (JOINT E)

Fig 1. Représentation schématique de la section des joints et de leur insertion dans la conduite. Pour chaque joint, l'intérieur de la conduite est situé en bas de la figure.

Simplified view showing the section of gaskets and their introduction into the pipeline. For each gasket, the internal side of the pipeline is in the lower part of the figure.

sence de défauts de surface, présence d'un encrassement résiduel.

\section{Au microscope électronique à balayage}

Les joints de 20 semaines et les joints neufs font l'objet d'une observation en microscopie électronique à balayage. Pour chaque joint, un échantillon de $0,5 \mathrm{~cm}$ de longueur est prélevé au hasard en le découpant avec des outils stérilisés. Les échantillons sont fixés dans une solution de glutaraldéhyde $3 \%(\mathrm{v} / \mathrm{v})$ dans du tampon cacodylate 0,2 mol. $\mathrm{L}^{-1} \mathrm{pH} 7,4$ pendant 1 heure à $4{ }^{\circ} \mathrm{C}$, puis rincés 3 fois pendant 10 minutes dans le tampon, et enfin déshydratés progressivement : 3 fois 10 minutes dans l'éthanol $70 \%(\mathrm{v} / \mathrm{v}), 3$ fois 10 minutes dans l'éthanol $95 \%(\mathrm{v} / \mathrm{v}), 3$ fois 10 minutes dans l'éthanol $100 \%$ à température ambiante. Ils sont séchés à l'air et métallisés à l'or (Jeol JFC1100E, Japon) pendant 420 
Tableau II. Procédures de nettoyage.

Cleaning operations.

\begin{tabular}{lllcrr}
\hline & $\begin{array}{c}\text { Intervalle entre } \\
\text { deux procédures }\end{array}$ & \multicolumn{1}{c}{ Produits } & $\begin{array}{c}\text { Concentration } \\
(\mathrm{v} / \mathrm{v})\end{array}$ & Température & $\begin{array}{r}\text { Durée } \\
(\text { min })\end{array}$ \\
\hline $\begin{array}{l}\text { Nettoyage } \\
\text { intermédiaire }\end{array}$ & $\begin{array}{l}12 \text { heures de } \\
\text { production au } \\
\text { maximum }\end{array}$ & $\begin{array}{l}\text { Soude } \\
\text { eau }\end{array}$ & $1,8 \%$ & $76^{\circ} \mathrm{C}$ & 46 \\
$\begin{array}{l}\text { Nettoyage } \\
\text { complet }\end{array}$ & $\begin{array}{l}\text { Après 500 000 litres } \\
\text { de production* }\end{array}$ & $\begin{array}{l}\text { Soude, } \\
\text { eau, } \\
\text { acide nitrique, } \\
\text { eau }\end{array}$ & $1 \%$ & $12^{\circ} \mathrm{C}$ & 6 \\
& & 1,8 & $76^{\circ} \mathrm{C}$ & 46 \\
& & $12^{\circ} \mathrm{C}$ & 6 \\
\hline
\end{tabular}

* Correspond à un total d'environ 17 heures de production.

* Corresponds to approximately 17 hours of production.

secondes, sous une tension de $10 \mathrm{kV}$, puis observés au microscope électronique à balayage (Jeol 5200 , Japon), sous une tension de $20 \mathrm{kV}$. Pour chaque joint, on observe la surface de la zone directement exposée aux fluides circulants, et la surface de la zone en contact imparfait avec le métal de la conduite. La face la plus externe du joint est utilisée pour fixer l'échantillon sur le support.

\section{Mesure de la contamination microbienne résiduelle}

\section{Traitement aux ultrasons pour détacher les cellules}

La mesure de la contamination microbienne résiduelle est effectuée après détachement dans une cuve à ultrasons (Delta 220, Deltasonic, Meaux, France) selon le protocole de Leriche et Carpentier (1995), non bactéricide sur des biofilms cultivés au laboratoire. Le détachement a lieu dans $100 \mathrm{~mL}$ (joints A, E, P) ou $250 \mathrm{~mL}$ (joint C, découpé aseptiquement en une quinzaine de morceaux) d'une solution neutralisante (peptone bactériologique neutre $10 \mathrm{~g} . \mathrm{L}^{-1}$, Tween $8040 \mathrm{~g} . \mathrm{L}^{-1}$, lécithine de soja 4 g.L $\mathrm{L}^{-1}$, histidine $1 \mathrm{~g} . \mathrm{L}^{-1}$, lauryl sulfate de sodium $4 \mathrm{~g} . \mathrm{L}^{-1}$, thiosulfate de sodium 17 g. $\mathrm{L}^{-1}$, extrait de viande 5 g.L. $\mathrm{L}^{-1}$, glycine 2 g.L. $\mathrm{L}^{-1}, \mathrm{pH} 7,0$ à $25^{\circ} \mathrm{C}$ ) dans laquelle le joint a été préalablement immergé pendant 5 minutes à $40^{\circ} \mathrm{C}$. Cette solution est préparée en se référant à l'annexe C de la norme AFNOR T 72-150
(Anonyme, 1987) et aux travaux de Van de Meyer et al (1993). L'étude de la contamination microbienne des joints entre dans le cadre d'une étude globale de la contamination microbienne après nettoyage, et éventuellement désinfection, de nombreux matériaux utilisés dans l'équipement et les locaux de l'industrie agro-alimentaire. C'est pourquoi le traitement de détachement par ultrasons est réalisé dans une solution neutralisante, commune à tous les cas étudiés, même si son emploi ne se justifie pas ici puisque les joints n'ont pas été en contact avec des désinfectants. Après le traitement de détachement, les joints sont rincés par $50 \mathrm{~mL}$ de tryptone-sel (AES Laboratoire, Combourg, France) et inclus dans la gélose, de façon à vérifier l'efficacité du traitement de détachement des micro-organismes.

\section{Dénombrement sur milieu gélosé}

Le dénombrement microbien sur milieu gélosé est effectué par ensemencement dans la masse, soit dans la gélose au lait (peptone de caséine 5 g.L $\mathrm{L}^{-1}$, extrait de levure $2,5 \mathrm{~g} . \mathrm{L}^{-1}$, glucose $1 \mathrm{~g} . \mathrm{L}^{-1}$, lait écrémé en poudre $1 \mathrm{~g} . \mathrm{L}^{-1}$, agar $15 \mathrm{~g} . \mathrm{L}^{-1}$ ) (Anonyme, 1985), soit dans la gélose Wilkins Chalgren (Oxoid Unipath, Dardilly, France) (tryptone 10 g. $\mathrm{L}^{-1}$, peptone de gélatine 10 g. $\mathrm{L}^{-1}$, extrait de levure 5 g. $\mathrm{L}^{-1}$, glucose 1 g.L $\mathrm{L}^{-1}$, chlorure de sodium 5 g.L $\mathrm{L}^{-1}$, L-arginine 1 g.L. $\mathrm{L}^{-1}$, pyruvate de sodium $1 \mathrm{~g} . \mathrm{L}^{-1}$, ménadione $0,5 \mathrm{mg} . \mathrm{L}^{-1}$, hémine $5 \mathrm{mg} \cdot \mathrm{L}^{-1}$, agar $10 \mathrm{~g} . \mathrm{L}^{-1}$ ) (Wilkins et Chalgren, 1976). Les ufc (unités formant colonie) sont comptées après une incubation de 72 heures à $30^{\circ} \mathrm{C}$ dans les deux cas. 


\section{Dénombrement par la méthode du nombre le plus probable (NPP)}

Le protocole utilisé est adapté de celui proposé par Gay et al (1996). On utilise deux microplaques de 96 puits pour chaque dilution (une seule micro-plaque par dilution pour l'essai de 20 semaines), chaque puits contenant $200 \mu \mathrm{L}$ de bouillon Wilkins Chalgren et $50 \mu \mathrm{L}$ de suspension bactérienne. L'incubation est de 72 heures à $30^{\circ} \mathrm{C}$. Le NPP est calculé à partir de trois dilutions successives à l'aide du logiciel NPP-INRALGHPA, appliquant les équations de Maul (1982). Cette méthode a été utilisée pour les joints de 4,16 et 20 semaines.

\section{Dénombrement direct des cellules colorées à l'orangé d'acridine}

Les solutions utilisées ont été préalablement filtrées à l'aide de membranes en acétate de cellulose ayant des pores de $0,22 \mu \mathrm{m}$ de diamètre (Millipore, St Quentin Yvelines, France). Un volume de $1 \mathrm{~mL}$ de la suspension bactérienne est filtré sur une membrane noire en polycarbonate ayant des pores de $0,22 \mu \mathrm{m}$ de diamètre (Nuclepore $13 \mathrm{~mm}$ de diamètre, Schumacher DMF, Gonesse, France). La membrane, maintenue dans le système de filtration, est recouverte d'une solution de Tris- $\mathrm{HCl} 50 \mathrm{mmol}^{-1}$, EDTA $5 \mathrm{mmol}$. $\mathrm{L}^{-1} \mathrm{pH} 8,0$, filtrée après 5 minutes de contact, puis recouverte par une solution d'orangé d'acridine $0,1 \%(\mathrm{~m} / \mathrm{v})$ (Rhône-Poulenc, VillersSaint-Paul, France) filtrée après 5 minutes de contact, puis rincée par filtration de $5 \mathrm{~mL}$ d'eau distillée. La membrane est ensuite montée sur lame de verre et observée avec un microscope à épifluorescence avec le filtre d'excitation KP500 et le filtre d'arrêt LP520 (Carl Zeiss, Le Pecq, France). Un minimum de 300 cellules sont comptées. Cette méthode a été appliquée pour des joints de 4 et 16 semaines.

\section{Identification des genres microbiens isolés}

Pour chaque joint, une boîte de chacun des deux milieux gélosés contenant si possible 30 ufc est sélectionnée. Les colonies sont dénombrées selon leur aspect. Les caractéristiques principales (morphologie de la cellule, coloration de Gram, type respiratoire, catalase, oxydase, mobilité à l'état frais) sont recherchées sur au maximum cinq colo- nies d'aspect identique, de façon à vérifier que celles-ci appartiennent au même genre bactérien.

\section{RÉSULTATS ET DISCUSSION}

\section{Observation de l'usure et de l'encrassement des joints}

\section{A l'œil nu}

A l'état neuf, les joints étant constitués du même matériau, ils ne diffèrent que par leur forme et leurs dimensions (fig 1). La surface est visuellement lisse pour les joints A (ou $P$ ) et $C$. Le joint $E$, dont l'état de surface est légèrement strié, se distingue notamment par sa petite taille et par sa forme relativement complexe. Il est probable que son mode de fabrication soit différent de celui employé pour les autres types de joints, ce qui pourrait expliquer la présence de stries à sa surface. Dès la quatrième semaine d'utilisation, les joints présentent des signes d'usure et d'encrassement, sur la face exposée aux fluides et sur les faces en contact avec le métal, qui s'accentuent au cours du temps. Seule la face la plus externe des joints ne présente ni signe d'usure ni encrassement après 20 semaines d'utilisation. Les déformations observées semblent dues à l'écrasement des faces du joint en contact avec le métal. Dès 4 semaines, la jonction entre celles-ci et la face exposée aux fluides est marquée par des stries parallèles à la circonférence interne, soulignées par des dépôts blancs, principalement sur les joints $\mathrm{A}$ et C. A partir de 16 semaines, on observe dans certains cas une crevasse (jusqu'à $1 \mathrm{~mm}$ de largeur et de profondeur, jusqu'à $3 \mathrm{~cm}$ de longueur) au niveau de cette jonction. Ces signes de détérioration physique visibles à l'œil nu sur les joints en EPDM correspondent à ceux déjà décrits sur des joints en polymère de type nitrile (Czechowski, 1990 ; Austin et Bergeron, 1995) et se produisent bien que les limites d'utilisation préconisées pour l'EPDM dépassent largement les valeurs des paramètres mis en cuvre lors des phases 
de production et de nettoyage de l'étude (Daufin et al, 1981). Or, le temps d'utilisation de 20 semaines ne représente que $1 / 5^{\mathrm{e}}$ de la durée d'utilisation maximale des joints, puisque ceux-ci ne sont habituellement remplacés que tous les 2 ans, en absence de fuite. Les joints sont donc altérés pratiquement dès le début de leur implantation.

Toute la face du joint $C$ exposée aux fluides présente une forte usure, avec un relief apparent, accompagnée d'un dépôt blanc abondant, qui résulte très probablement de la précipitation de phosphate de calcium, dont la solubilité diminue quand la température augmente et qui est le principal composant de l'encrassement minéral des échangeurs de chaleur (Bouman et al, 1982). Le nettoyage complet, qui précède le prélèvement des joints, comprend notamment un traitement à l'acide nitrique ; le dépôt de phosphate de calcium montre que ce traitement n'est pas suffisant pour éliminer entièrement l'encrassement minéral sur les joints placés dans la zone de chambrage.

\section{Au microscope électronique à balayage}

Les figures 2 à 6 présentent les joints observés en microscopie électronique à balayage

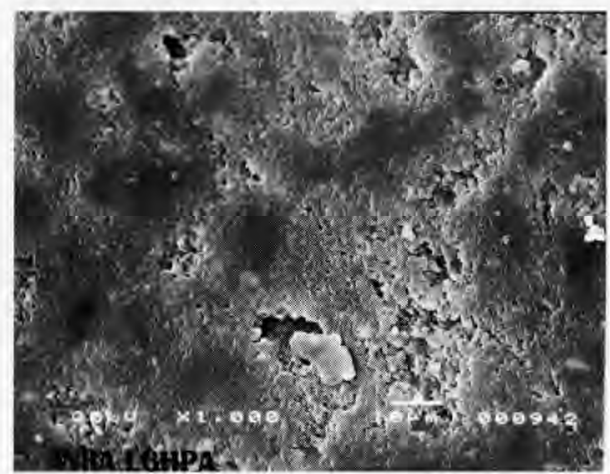

Fig 2. Photographie au microscopie électronique à balayage d'un joint avant son implantation dans le circuit.

Scanning electron microphotograph showing the gasket before introduction into the pipeline. classés dans l'ordre de parcours du lait. A l'état neuf, le matériau visuellement lisse montre au niveau microscopique une surface sans dépôt ni crevasse, mais on observe en certains points l'arrachement de quelques particules en surface (fig 2). Cette altération pourrait être provoquée lors du stockage précédant l'utilisation (Daufin et al, 1981). On retrouve à l'échelle microscopique les stries visibles à l'œil nu sur le joint de sortie d'écrémeuse neuf $\mathrm{E}$ (fig 5a).

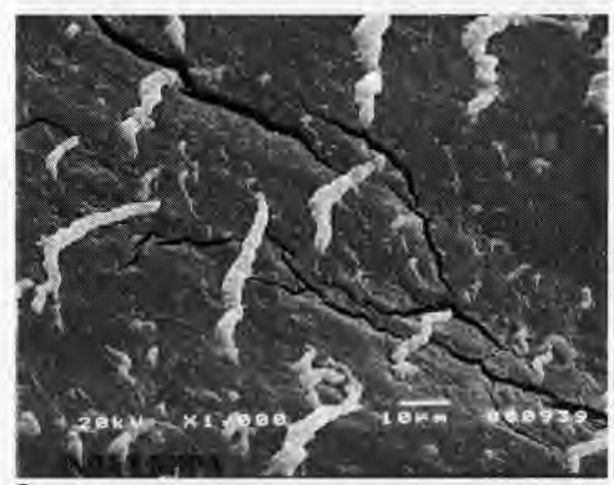

a

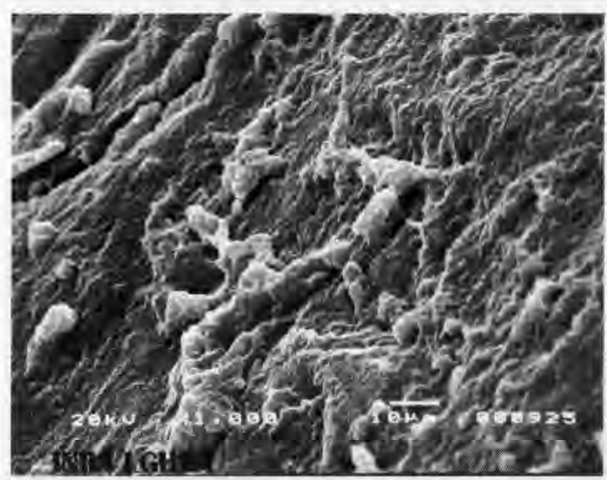

b

Fig 3. Photographie au microscopie électronique à balayage du joint $\mathrm{A}$ (arrivée du lait cru) après 20 semaines d'utilisation, au niveau de la face exposée aux fluides (a) et au niveau de la jonction entre la face exposée aux fluides et la zone en contact avec le métal (b).

Scanning electron microphotograph showing the 20-week gasket A (raw milk inlet), in the area exposed to fluids (a) and at the junction-area between the face exposed to fluids and the face in contact with metal (b). 

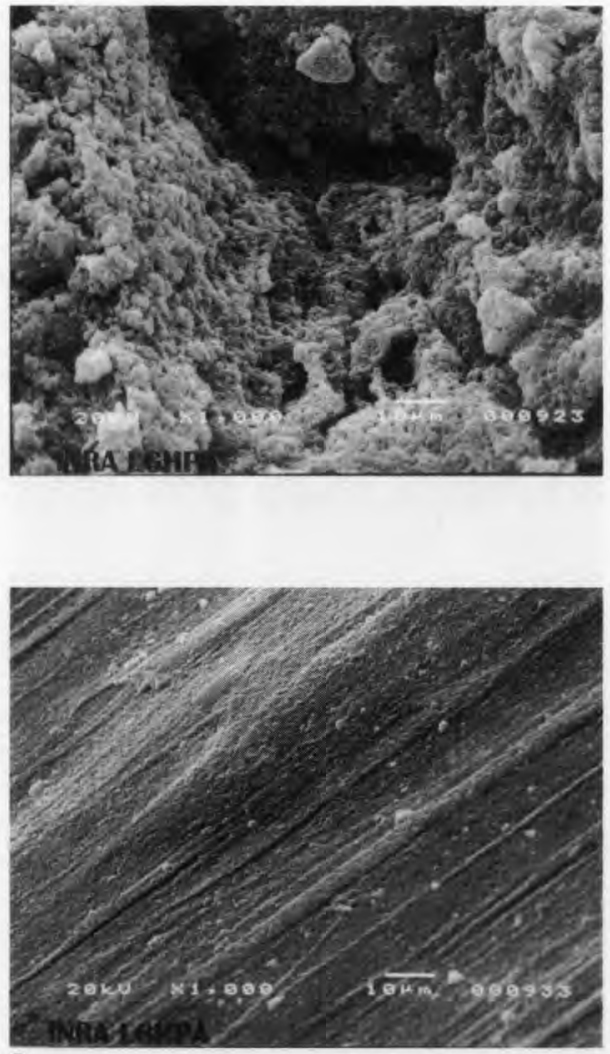

a

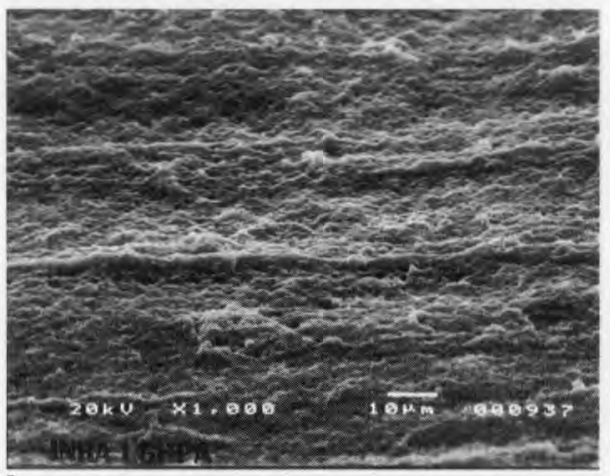

b
Fig 4. Photographie en microscopie électronique à balayage du joint $\mathrm{C}$ (chambrage) après 20 semaines d'utilisation, au niveau de la face exposée aux fluides.

Scanning electron microphotograph showing the 20-week gasket $C$ (during pasteurization), in the area exposed to fluids.

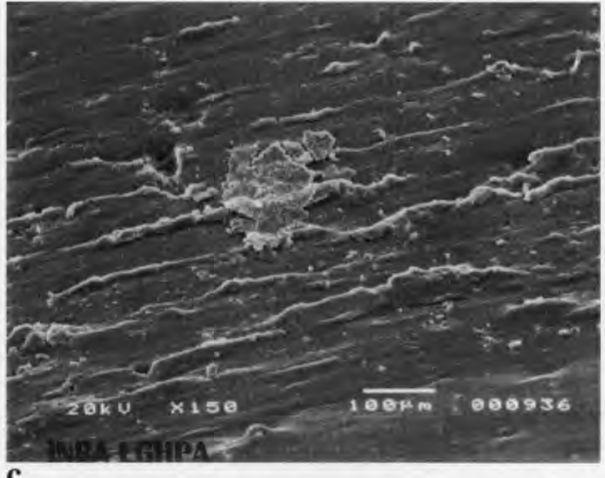

c

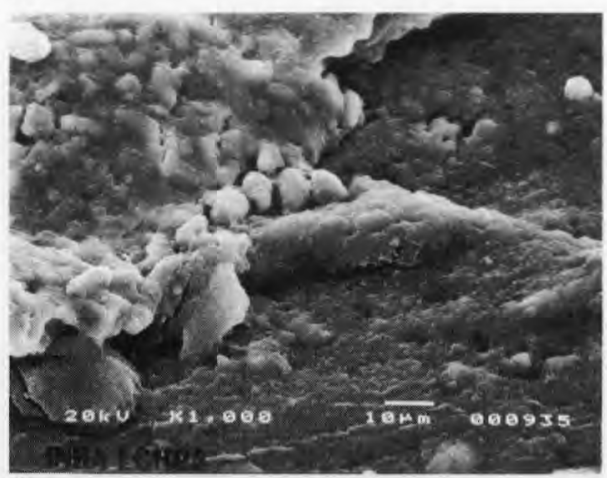

d

Fig 5. Photographie en microscopie électronique à balayage du joint $\mathrm{E}$ (sortie de l'écrémeuse) au niveau de la face exposée aux fluides avant son implantation (a) et après 20 semaines d'utilisation (b), au niveau de la face en contact avec le métal après 20 semaines d'utilisation avec un grossissement par 150 (c) et par 1000 (d).

Scanning electron microphotograph showing gasket E (separator outlet), in the area exposed to fluids for the new gasket (a) and for the 20-week gasket (b), and in the area in contact with metal for the 20-week gasket E, at magnifications of 150 times (c) and 1000 times (d). 


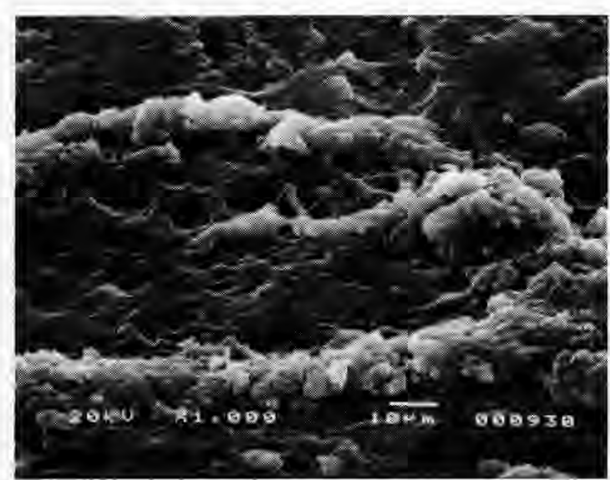

Fig 6. Photographie au microscopie électronique à balayage de la surface du joint $\mathrm{P}$ (sortie du lait pasteurisé) après 20 semaines d'utilisation, au niveau de la face exposée aux fluides.

Scanning electron microphotograph showing 20 week gasket $P$ (pasteurized milk outlet) in the area exposed to fluids.

Après 20 semaines d'utilisation, un encrassement résiduel plus ou moins épais recouvre toutes les surfaces observées. Seule la face du joint A exposée aux fluides laisse affleurer la surface du matériau (fig 3a), ce qui permet de visualiser un réseau de crevasses radiales, dont la largeur est de l'ordre de $1 \mu \mathrm{m}$. Pour le même joint, au niveau de la zone de jonction entre la face exposée aux fluides et la zone de contact joint-métal (fig 3b), la surface du matériau est complètement recouverte par un encrassement d'aspect organique. Un dépôt continu d'aspect comparable est observé sur les faces exposées aux fluides des joints $E$ (fig 5b) et $\mathbf{P}$ (fig 6). En revanche, l'aspect du dépôt continu qui recouvre la face exposée aux fluides du joint $C$ (fig 4) est différent : on retrouve l'encrassement minéral déjà visible à l'œil nu, constitué de particules élémentaires ne dépassant pas quelques micromètres de diamètre. Celles-ci peuvent correspondre aux particules plus ou moins sphériques, de 0,2 à $2 \mu \mathrm{m}$ de diamètre, constituant le dépôt de phosphate de calcium décrit sur les plaques d'échangeur de chaleur (Bouman et al, 1982 ; Driessen et al, 1984). Les zones du joint $C$ en contact avec le métal présentent un dépôt proche de celui observé sur les autres joints (résultat non présenté).

Dans plusieurs cas, on observe des amas de résidus formant des filaments orientés selon une direction parallèle à la circonférence, sur les joints $A$ (fig $3 a$ ), E (fig $5 c$ ) et P (fig 6). L'absence de lien apparent entre les crevasses et l'encrassement, observée sur le joint $\mathrm{A}$, a déjà été décrite (Czechowski, 1990). Contrairement à cet auteur, qui utilise la microscopie à épifluorescence, nous ne pouvons pas distinguer si les amas abritent des micro-organismes : les particules globulaires observées sur le joint E (fig $5 \mathrm{~d}$ ), sont de taille trop importante pour être identifiées comme des cellules microbiennes et les petits filaments longs de 1 à $5 \mu \mathrm{m}$ visibles sur le joint A (fig 3a) ne sont pas forcément des microorganismes. Excepté pour le joint $\mathrm{C}$, l'encrassement résiduel est plus abondant dans la zone de contact jointmétal que sur la face du joint exposée aux fluides, comme cela a déjà été décrit (Czechowski, 1990 ; Austin et Bergeron, 1995). Ceci est attribué au fait que la zone de contact joint-métal est difficilement accessible aux solutions de nettoyage.

\section{Contamination microbienne résiduelle}

La technique de détachement s'applique à toute la surface du joint. Or, il n'est pas possible de connaître la superficie exacte du joint qui est contaminée. Les observations en microscopie électronique montrent qu'elle ne se limite pas à la superficie directement exposée aux fluides, variable entre les joints (fig 1), puisque l'encrassement microbien peut s'implanter dans des zones de contact joint-métal. En revanche, la face externe des joints, visiblement sèche et non encrassée en période de production, n'est pas favorable à la croissance microbienne. Par conséquent, les résultats des dénombrements 
(tableau III) ne peuvent être exprimés par unité de surface. Ils sont toutefois ramenés à $1 \mathrm{~cm}$ de périmètre interne du joint de manière à tenir compte des différences de diamètre entre les joints décrites dans la figure 1.

L'inclusion des joints dans la gélose après détachement et rinçage n'a mis en évidence des micro-organismes non détachés que dans deux cas : le joint A de 1 semaine et le joint $\mathrm{C}$ de 16 semaines. Le premier cas indique un détachement défectueux, aucun micro-organisme n'ayant été détecté dans la solution de détachement. Dans le second cas, les chiffres obtenus sont homogènes avec les autres dénombrements. Il est donc fort pro- bable que les micro-organismes non détachés soient très fortement minoritaires par rapport à la population détachée.

\section{Comparaison des méthodes de dénombrement}

La gélose au lait constitue notre milieu de référence. Le milieu Wilkins Chalgren contient notamment des intermédiaires métaboliques, tels que le pyruvate et l'arginine, qui peuvent compenser les pertes cellulaires éventuelles liées au stress. D'après Wilkins et Chalgren, le pyruvate agit comme une catalase (la réaction de dégradation du péroxyde d'hydrogène n'est pas aussi rapide

Tableau III. Logarithme du nombre d'unités microbiennes par centimètre de périmètre interne de joint, obtenu par les différentes méthodes de dénombrement de la solution de détachement.

Logarithm of the number of microbial units per centimeter of the internal perimeter of gaskets, calculated from the different enumeration methods applied to the detachment solution.

\begin{tabular}{|c|c|c|c|c|c|}
\hline & & $\begin{array}{l}\text { Gélose } \\
\text { au lait }\end{array}$ & $\begin{array}{c}\text { Gélose } \\
\text { Wilkins } \\
\text { Chalgren }\end{array}$ & $\begin{array}{c}\text { Bouillon } \\
\text { Wilkins } \\
\text { Chalgren } \\
\text { (NPP) }\end{array}$ & $\begin{array}{l}\text { Coloration } \\
\text { à l'orangé } \\
\text { d'acridine }\end{array}$ \\
\hline \multirow[t]{4}{*}{ Joint A } & $1 *$ & $<0,2$ & $<0,2$ & & \\
\hline & 4 & 0,8 & 1,2 & 1,1 & 6,2 \\
\hline & 16 & 2,3 & 2,5 & 2,7 & 6,2 \\
\hline & 20 & 2,3 & 2,5 & 2,4 & \\
\hline \multirow[t]{4}{*}{ Joint C } & 1 & $<-0,2$ & $<-0,2$ & & \\
\hline & 4 & 1,0 & 0,2 & 0,3 & 6,8 \\
\hline & 16 & 2,6 & 2,7 & 3,0 & 6,6 \\
\hline & 20 & 0,4 & 0,8 & 0,7 & \\
\hline \multirow[t]{4}{*}{ Joint E } & 1 & 1,8 & 1,8 & & \\
\hline & 4 & 2,0 & 1,9 & 1,5 & 5,8 \\
\hline & 16 & 0,2 & $<0,2$ & 0,7 & 6,7 \\
\hline & 20 & 2,9 & 3,1 & 3,1 & \\
\hline \multirow[t]{4}{*}{ Joint $\mathrm{P}$} & 1 & 0,2 & $<0,2$ & & \\
\hline & 4 & $<0,2$ & $<0,2$ & $<0,1$ & 6,1 \\
\hline & 16 & 0,2 & $<0,2$ & 0,1 & 6,2 \\
\hline & 20 & 3,0 & 3,2 & 3,3 & \\
\hline
\end{tabular}

* Durée d'utilisation du joint, en semaines.

* Time of use of gaskets, in weeks. 
que celle obtenue avec la catalase mais le pyruvate présente l'avantage de pouvoir être autoclavé avec le milieu) et il convient donc pour le dénombrement de bactéries ayant subi un stress thermique, tout particulièrement pour les staphylocoques (Martin et al, 1976). La méthode du NPP a été utilisée ici pour éviter le stress thermique qui peut éventuellement se produire par la mise en contact avec la gélose en surfusion (Mossel et Van Netten, 1984). Cette méthode présente également l'avantage d'avoir un seuil de détection plus faible (ici, une bactérie dans $4,8 \mathrm{~mL}$ ).

Pour chaque joint, la comparaison des résultats obtenus avec les trois méthodes de dénombrement des bactéries cultivables (tableau III) ne montre globalement aucune différence significative. D'une part, l'utilisation du milieu Wilkins Chalgren n'apporte pas d'amélioration détectable par rapport à la gélose au lait. Soit la majorité des cellules viables sont ici cultivables sur gélose au lait, ce qui signifierait que la gélose au lait les mettrait toutes en évidence, soit les additifs contenus dans le milieu Wilkins Chalgren ne suffisent pas pour rendre cultivables des cellules viables non mises en évidence sur gélose au lait. D'autre part, la culture en bouillon (méthode du NPP) n'apporte pas d'amélioration détectable par rapport au dénombrement en gélose. Cela n'est pas surprenant ici, les micro-organismes cultivables n'étant a priori pas affectés par la température de la gélose en surfusion, puisqu'ils supportent des températures beaucoup plus élevées dans la situation industrielle décrite.

On observe dans tous les cas une forte différence (au moins 3,5 puissances de 10) entre le dénombrement de la population cultivable et le dénombrement de la population totale (colorée à l'orangé d'acridine). Cette différence n'étant vraisemblablement pas due à une mortalité liée au traitement par les ultrasons (Leriche et Carpentier, 1995), elle indique que la plus grande partie des cellules microbiennes non détachées par les procédures de nettoyage correspond à des cellules inactivées par ces mêmes procédures, mortes et/ou viables non cultivables dans les conditions décrites dans l'étude. La contamination d'une surface après plusieurs cycles encrassement/opérations d'hygiène résulte d'une accumulation progressive de micro-organismes qui ne peuvent être ni totalement détachés ni totalement inactivés (Dunsmore, 1981). Le fait que la valeur de la population totale soit très supérieure à la valeur de la population cultivable indique que l'inactivation des microorganismes est plus efficace que le détachement de ceux-ci. Compte tenu du fait que l'on trouve $10^{6}$ cellules $\mathrm{cm}^{-1}$ de périmètre de joint, il apparaît évident que les amas de souillures observés par microscopie électronique à balayage abritent des microorganismes.

\section{Comparaison des joints selon leur emplacement}

La valeur de la population totale n'est globalement pas affectée par l'emplacement des joints. Les joints situés à l'entrée et à la sortie du circuit étudié ne montrent aucune différence. De même, Austin et Bergeron (1995) observent un encrassement microbien, aussi bien dans la zone du lait pasteurisé que dans la zone du lait cru, alors que Czechowski (1990) rapporte un pourcentage "généralement plus élevé » de joints contaminés dans la zone du lait cru.

Les joints ne se distinguent pas non plus en termes de cellules cultivables : le niveau maximal obtenu pour les quatre emplacements est du même ordre de grandeur.

\section{Évolution dans le temps de la population microbienne}

La population totale est globalement stable entre 4 et 16 semaines. D'après Czechowski (1990), une durée d'implantation de 6 semaines est nécessaire pour détecter par 
épifluorescence une contamination résiduelle après nettoyage sur tous les joints implantés.

La population des cellules cultivables est plus élevée à 16 ou 20 semaines qu'à 1 et 4 semaines. Deux points sortent de la tendance générale : le joint $\mathrm{C}$ de 20 semaines et le joint $\mathrm{E}$ de 16 semaines montrent une contamination très faible par rapport aux autres joints pour les mêmes durées d'utilisation. Or, dans les deux cas, le traitement par ultrasons a entraîné la libération d'une coloration noire dans la solution de détachement. Il s'agit vraisemblablement d'un relargage de carbone (Daufin et al, 1981), dont on peut supposer qu'il affecte sensiblement la croissance des bactéries lors du dénombrement sur milieu gélosé ou en bouillon, alors que le nombre total de cellules sur le joint $\mathrm{E}$ de 16 semaines est du même ordre de grandeur que celui des autres joints pour la même durée d'utilisation (valeur non déterminée dans le cas du joint $C$ de 20 semaines).

La population cultivable atteint un ordre de grandeur de $10^{3} \mathrm{ufc} . \mathrm{cm}^{-1}$ de périmètre soit à 16 semaines, soit à 20 semaines. Le prélèvement des joints devant avoir lieu avant le passage d'eau à $95^{\circ} \mathrm{C}$ pendant 110 minutes, nous ne savons pas dans quelle proportion cette population cultivable est réduite avant la reprise de la production. Cependant, une partie de cette population survit puisque l'encrassement microbien cultivable augmente au cours du temps.

Cela n'est pas surprenant puisque la thermorésistance des bactéries peut être augmentée par leur adhésion à un support (Frank et Koffi, 1990), par leur association étroite avec des souillures organiques dans les anfractuosités (Lelieveld, 1996) et par leur localisation entre le joint et le métal (Pfeifer et Kessler, 1995). Ces derniers montrent que la thermorésistance des spores de Bacillus cereus est considérablement augmentée quand elles sont confinées dans une zone de contact joint-métal. Ils attribuent ce résultat à la réduction de l'activité de l'eau provoquée dans cette zone par le chauffage. Dans une enceinte close chauffée, l'eau s'évapore puis devient sèche et surchauffée, ce qui correspond à une baisse de l'activité de l'eau. Les mêmes auteurs montrent par ailleurs que la thermorésistance des spores de Bacillus cereus augmente lorsque l'activité de l'eau diminue, avec une résistance maximale lorsque $\mathrm{a}_{\mathrm{w}}=0,4$ (Pfeifer et Kessler, 1994). La même observation peut être faite pour des bactéries non sporulées, notamment Micrococcus luteus (Rademacher, 1993).

Dunsmore (1981) a montré par simulation de cycles d'encrassement et de nettoyage en place que l'utilisation d'un protocole de nettoyage et de désinfection complet n'empêche pas une augmentation au cours du temps, lente mais régulière, de la population microbienne cultivable : après 36 cycles d'encrassement et de nettoyage en place, correspondant à quelques semaines d'utilisation, il compte $10^{2}$ ufc $\mathrm{cm}^{-2}$ sur les surfaces en contact avec le lait après détachement par écouvillonnage. Cette valeur obtenue expérimentalement dans des conditions très différentes des nôtres permet d'aboutir à des conclusions comparables.

\section{Genres microbiens isolés}

Le tableau IV décrit les genres isolés des joints de 16 et 20 semaines. Il indique également la proportion de chaque genre par rapport à la flore totale isolée de chaque joint. Nous n'observons pas de genre spécifique à un milieu, c'est pourquoi le tableau est établi à partir des résultats cumulés des deux milieux.

On note peu de différences entre les flores de 16 et 20 semaines. On observe toutefois la présence du genre Bacillus uniquement pour les joints de 16 semaines, où il est codominant, et la présence du genre $P$ seudomonas uniquement à 20 semaines, mais très minoritaire. Les genres dominants à 20 semaines sont déjà présents à 16 semaines. 
On n'observe pas de flore spécifique à une localisation de joint.

Tous les genres identifiés parmi les bactéries cultivables font partie de la flore du lait cru conservé à une température inférieure à $4{ }^{\circ} \mathrm{C}$ (Lewis et Gilmour, 1987 ; Burgess et al, 1994), ce qui est en accord avec les études démontrant que la contamination des matériaux des conduites a pour origine la contamination du lait (Langeveld et al, 1995). On observe une nette dominance des genres Gram positifs, comme dans le lait cru. Or, plusieurs études montrent que l'adhésion des genres Gram négatifs est supérieure à l'adhésion des genres Gram positifs sur ces matériaux (Speers et Gilmour, 1985 ; Criado et al, 1994), ce qui entraîne la formation d'un encrassement microbien essentiellement constitué de genres Gram négatifs avant nettoyage (Lewis et Gilmour, 1987). En revanche, l'utilisation de la microscopie électronique à transmission pour observer une coupe du biofilm formé sur un joint mis en place pendant 4 semaines a permis à Austin et
Bergeron (1995) de constater que des bactéries Gram négatives altérées constituent la base du biofilm, ce qui est en accord avec leur plus grande aptitude à adhérer citée précédemment, alors que des bactéries Gram positives en constituent la face supérieure et ne présentent aucun signe d'altération. Cela peut expliquer que les genres retrouvés parmi les bactéries cultivables dans notre étude soient essentiellement des bactéries Gram positives. Par ailleurs, les bactéries Gram négatives sont généralement considérées comme incapables de survivre à la pasteurisation (Burgess et al, 1994 ; Broens et al, 1996). La circulation d'eau à $95^{\circ} \mathrm{C}$ qui suit le nettoyage pourrait a fortiori les empêcher de s'implanter durablement.

La stabilité globale de la flore au cours du temps et entre les quatre emplacements indique un encrassement qualitativement homogène. On n'observe notamment pas de différence d'implantation des micro-organismes en fonction de la température. Langeveld et al (1995), ont pourtant montré que des bactéries inoculées simultanément à

Tableau IV. Identification partielle des genres isolés des joints de 16 et 20 semaines, et pourcentage de chaque genre par rapport à la contamination totale de chaque joint.

Partial identification of the microbial types detached from 16-week and 20-week gaskets. Percentage of each type relative to the total contamination of each gasket.

\begin{tabular}{llllr}
\hline & 16 semaines & \multicolumn{3}{l}{20 semaines } \\
\hline \multirow{2}{*}{ Joint A } & Bacillus spp & $52 \%$ & Micrococcus $\mathrm{spp}$ & $85 \%$ \\
& Micrococcus $\mathrm{spp}$ & $35 \%$ & Staphylococcus $\mathrm{spp}$ & $13 \%$ \\
& Staphylococcus $\mathrm{spp}$ & $13 \%$ & Pseudomonas $\mathrm{spp}$ & $2 \%$ \\
\multirow{3}{*}{ Joint C } & Staphylococcus $\mathrm{spp}$ & $58 \%$ & Micrococcus $\mathrm{spp}$ & $100 \%$ \\
& Bacillus $\mathrm{spp}$ & $42 \%$ & & \\
\multirow{2}{*}{ Joint P } & Bacillus $\mathrm{spp}$ & $100 \%$ & Staphylococcus $\mathrm{spp}$ & $50 \%$ \\
& & & Micrococcus $\mathrm{spp}$ & $45 \%$ \\
& & & Pseudomonas $\mathrm{spp}$ & $5 \%$ \\
\multirow{3}{*}{ Joint $\mathrm{E}$} & - & & Staphylococcus $\mathrm{spp}$ & $90 \%$ \\
& & & Micrococcus $\mathrm{spp}$ & $10 \%$ \\
\hline
\end{tabular}


faible concentration dans le lait à l'entrée d'un pasteurisateur adhèrent à l'acier inoxydable dans des zones différentes du circuit, en fonction de la température de la paroi (Escherichia coli entre 22 à $49^{\circ} \mathrm{C}$, Thermus thermophilus entre 67 et $83^{\circ} \mathrm{C}$ ). Il s'agit cependant de la contamination avant nettoyage. Ici, le traitement de nettoyage est appliqué de façon identique à tous les joints, alors que les conditions de production varient d'un point à un autre du circuit. L'homogénéité de la composition de la flore résiduelle permet donc de supposer que celle-ci serait essentiellement déterminée par l'aptitude des différentes souches à résister au nettoyage, plutôt que par leur aptitude à adhérer et/ou à former un biofilm.

\section{CONCLUSION}

Le caractère agressif des conditions de production et des procédures de nettoyage est mis en évidence par l'usure rapide et marquée des joints de la ligne de pasteurisation. Pourtant les procédures de nettoyage en place ne sont pas suffisantes pour empêcher l'établissement d'un encrassement résiduel minéral et/ou organique à la surface des joints, qui concerne non seulement la face en contact avec les fluides circulants, mais aussi les faces en contact imparfait avec le métal. La population microbienne cultivable résistant à la procédure de nettoyage, constituée essentiellement de quelques genres microbiens Gram positifs, augmente au cours du temps pour atteindre environ $10^{3}$ ufc par centimètre de périmètre de joint après 16 ou 20 semaines d'utilisation, aussi bien en amont qu'en aval du pasteurisateur. De plus, la population totale de cellules, cultivables et non cultivables, atteint $10^{6}$ cellules par centimètre de périmètre de joint. Or, il est très probable qu' une partie de la population non cultivable reste viable et augmente donc la population microbienne susceptible de provoquer une contamination du lait. L'objectif final étant la qualité hygiénique du lait traité, il est nécessaire de déterminer dans quelle mesure cette contamination résiduelle des joints augmente le risque d'avoir un produit non conforme à la législation.

\section{REMERCIEMENTS}

Les auteurs remercient chaleureusement D Chassaing pour la qualité de son assistance technique, I Reyns et N Petiot pour leur contribution active à la réalisation de l'étude sur le terrain. Leurs remerciements s'adressent également à I'Inra-LGHPA pour la microscopie électronique à balayage et pour le prêt du logiciel NPP, à UNIR et au ministère de la Recherche et de l'Enseignement supérieur pour le financement de cette étude.

\section{RÉFÉRENCES}

Anonyme (1985) Lait. Dénombrement des micro-organismes. Technique par comptage des colonies à $30^{\circ} \mathrm{C}$. Norme AFNOR V 04-016

Anonyme (1987) Antiseptiques et désinfectants utilisés à l'état liquide, miscibles à l'eau et neutralisables. Détermination de l'activité bactéricide. Méthode par dilution neutralisation. Norme AFNOR T 72 150

Austin JW, Bergeron G (1995) Development of bacterial biofilms in dairy processing lines. $J$ Dairy Res 3, 509-519

Bouman S, Lund DB, Driessen FM, Schmidt DG (1982) Growth of thermoresistant streptococci and deposition of milk constituents on plates of heat exchangers during long operating times. $J$ Food Prot 45, 806-813

Bourion F, Cerf O (1996) Disinfection efficacy against pure-culture and mixed-population biofilms of Listeria innocua and Pseudomonas aeruginosa on stainless steel, Teflon and rubber. Sci Aliment 16, 151-166

Broens E, Masolijn A, Martens F, Smith A, Dawson P, Richardson S, Pfaffli M (1996) Rapid method for the detection of post-pasteurisation contamination in pasteurised milk. In : Compte rendu de la quatrième conférence internationale ASEPT Sécurité alimentaire 96 (Amgar A, ed), ASEPT Editeur, Laval, 362

Burgess K, Heggum C, Walker S, Van Schothorst M (1994) Recommendations for the hygienic manufacture of milk and milk based products. Int Dairy Fed doc 292, 28-32 
Carpentier B, Cerf O (1993) Biofilms and their consequences, with particular reference to hygiene in the food industry. J Appl Bacteriol 75, 499-511

Criado MT, Suárez B, Ferreirós CM (1994) The importance of bacterial adhesion in the dairy industry. Food Technol February, 123-126

Curiel GJ, Hauser G, Peschel P, Timperley DA (1993) EHEDG update: hygienic equipment design criteria. Trends Food Sci Technol 4, 225-229

Czechowski MH (1990) Bacterial attachment to Buna$n$ gaskets in milk processing equipment. Aust J Dairy Technol 45, 113-114

Daufin G, Cowan CT, Flückiger E, Pagetti J, Wildbrett $G$ (1981) Rubber corrosion in the dairy processing industry. Int Dairy Fed doc 139, brochure 4

Driessen FM, De Vries J, Kingma F (1984) Adhesion and growth of thermoresistant streptococci on stainless steel during heat treatment of milk. $J$ Food Prot 47, 848-852

Dunsmore DG (1981) Bacteriological control of food equipment surfaces by cleaning systems. I. Detergent effects. J Food Prot 44, 15-20

Frank JF, Koffi RA (1990) Surface-adherent growth of Listeria monocytogenes is associated with increased resistance to surfactant sanitizers and heat. J Food Prot 53, 550-554

Gay M, Cerf O, Davey KR (1996) Significance of preincubation temperature and inoculum concentration on subsequent growth of Listeria monocytogenes at $14^{\circ} \mathrm{C}$. I Appl Bacteriol 81, 433-438

Kane DR, Middlemiss NE (1985) Cleaning chemicals. State of the knowledge in 1985. In: Fouling and cleaning in food processing (Lund D, Plett EA, Sandu C, eds) Madison, Wi, USA, 312-335

Karpinsky JL, Bradley RL (1988) Assessment of the cleanability of air-actuated butterfly valves. J Food Prot 51, 364-368

Langeveld LPM, van Monfort-Quasig RMGE, Weerkamp AH, Waalewijn R, Wever JS (1995) Adherence, growth and release of bacteria in a tube heat exchanger for milk. Neth Milk Dairy J 49, 207-220

Lelieveld HLM (1996) Protection of microorganisms in soil against thermal and chemical inactivation. In : Compte rendu de la quatrième conférence internationale ASEPT Sécurité Alimentaire 96 (Amgar A, ed), ASEPT Editeur, Laval, 337-343

Leriche V, Carpentier B (1995) Viable but nonculturable Salmonella typhimurium in single- and binary- species biofilms in response to chlorine treatment. J Food Prot 58, 1186-1191

Lewis SJ, Gilmour A (1987) Microflora associated with the internal surfaces of rubber and stainless steel milk transfer pipeline. J Appl Bacteriol 62, 327-333

Martin SE, Flowers RS, Ordal ZJ (1976) Catalase: its effect on microbial enumeration. Appl Environ Microbiol 32, 731-734

Maul A (1982) Définition d'une stratégie de prélèvement pour l'analyse microbiologique des eaux de surface. Thèse de doctorat de $3^{\mathrm{e}}$ cycle, Centre des sciences de l'environnement, Metz

Mossel DAA, Van Netten P (1984) Harmful effects of selective media on stressed micro-organisms. In : The revival of injured microbes (Andrew MHE, Russel AD, eds) Society for Applied Bacteriology, Symposium Series n ${ }^{\circ} 12$, Academic Press, London, 329-369

Pfeifer J, Kessler HG (1994) Effect of relative humidity of hot air on the resistance of Bacillus cereus spores. J Appl Bacteriol 77, 121-128

Pfeifer J, Kessler HG (1995) Heat resistance of Bacillus cereus spores located between seals and seal surfaces. I Food Prot 58, 1206-1210

Rademacher B (1993) Einfluß der Wasseraktivität auf die Hitzeinaktivierung vegetativer Mikroorganismen. In : Wissenschaftlicher Jahresbericht 1992 , Forschungszentrum für Milch und Lebensmittel, Weihenstephan, 171-172

Speers JGS, Gilmour A (1985) The influence of milk and milk components on the attachment of bacteria to farm dairy equipment surfaces. J Appl Bacteriol $59,325-332$

Van de Meyer A, Devleeschouwer MJ, Dony J (1993) Bactericidal activity of disinfectants on listeria. $J$ Appl Bacteriol 74, 480-483

Wilkins TD, Chalgren S (1976) Medium for use in antibiotic susceptibility testing of anaerobic bacteria. Antimicrob Agents Chemother 10, 926-928

Wirtanen G, Storgărds E, Tikkanen K, Mattila-Sandholm T (1996) Evaluation of the cleaning effect on rubber and stainless steel surfaces used in closed dairy and brewery equipment. In : Compte rendu de la quatrième conférence internationale ASEPT Sécurité alimentaire 96 (Amgar A, ed), ASEPT Editeur, Laval, 374 